\title{
RADIO AND OPTICAL ASTROMETRY
}

(Invited Paper)

\author{
C. M. WADE \\ National Radio Astronomy Observatory*, Green Bank, W.Va., U.S.A.
}

\begin{abstract}
Radio positional measurements have achieved an accuracy as high as that of optical astrometry, with uncertainties no greater than a few hundredths of an arc second in each coordinate. Declinations and relative right ascensions are determined absolutely. Since the principal sources of systematic error are different for radio and optical astrometry, radio measurements can be useful in the preparation of future fundamental catalogues.
\end{abstract}

\section{Introduction}

A quarter of a century ago, the most accurate measurements of radio source positions were uncertain by several minutes of arc. The quality of radio work has since improved to the point that it now is possible to make systematic positional measurements of small-diameter sources which are as accurate as the fundamental optical catalogues. Interferometric methods developed at the Mullard Radio Astronomy Observatory in Great Britain and at the National Radio Astronomy Observatory in the United States yield precise absolute declinations and right ascension differences. It still is necessary to use optical information to fix the origin of the right ascensions, since no object in the solar system is suitable for radio astrometry. In all other respects, the radio measurements are fundamental in the astrometric sense.

Radio astrometry has several important advantages in comparison with optical astrometry. The effects of atmospheric refraction are relatively unimportant. Large angles can be measured with about the same accuracy as small ones, so regional systematic errors are not a serious problem. Observations of a large number of sources well distributed on the sky can be analyzed simultaneously in a way that fixes most of the instrumental parameters along with the source positions, so the measurements are largely self-calibrating. The observations determine the radii of the diurnal circles of the sources, thus referring the declinations directly to the Earth's instantaneous axis of rotation. Most sources of small angular size are extragalactic, and a grid of fundamental positions which are not affected by proper motions can be established readily. Finally, the observations can be made rapidly, and analyzed entirely by electronic computers.

We shall consider here only the most recent fundamental radio interferometric measurements. Much excellent radio work has been done by reference to optically determined calibration positions, but such measurements are outside the scope of the present paper since they are essentially differential. We shall also ignore lunar occulta-

* Operated by Associated Universities, Inc., under contract with the National Science Foundation. 
tion measurements because their accuracy is limited by instrumental noise and the uncertainty of the lunar limb corrections.

\section{Fundamental Radio Measurements}

Radio interferometric techniques for absolute position measurements have been developed independently at the Mullard Radio Astronomy Observatory (Elsmore and Mackay, 1969; Smith, 1971; Ryle and Elsmore, 1973) and at the National Radio Astronomy Observatory (Wade, 1970; Brosche et al., 1973). Although the underlying principles of the measurements at the two observatories are much the same, the actual methods differ considerably because the instruments are quite dissimilar (Ryle, 1972; Hogg et al., 1969). The difference which has the greatest influence on method is in the baseline orientations. Some of the characteristics of the interferometers are summarized in Table I.

TABLE I

\begin{tabular}{lcc} 
Characteristics of the Mullard and NRAO interferometers \\
\hline & Mullard & NRAO \\
\hline & 8 & 3 \\
Number of antennas & $13 \mathrm{~m}$ & $26 \mathrm{~m}$ \\
Antenna diameter & $4.6 \mathrm{~km}$ & $2.7 \mathrm{~km}$ \\
Maximum baseline & $93^{\circ} 19^{\prime}$ & $62^{\circ} 02^{\prime}$ \\
Baseline azimuth & $52^{\circ} .2 \mathrm{~N}$ & $38^{\circ} .4 \mathrm{~N}$ \\
Latitude & $6 \mathrm{~cm}$ & $4+11 \mathrm{~cm}$ \\
Operating wavelength & $2^{\prime \prime} .7$ & $2^{\prime \prime} .9$ \\
Min. fringe spacing &
\end{tabular}

The accuracy of both instruments is limited primarily by random fluctuations in the signal paths through the troposphere (and to a lesser extent, through the ionosphere). The effect is analogous to optical scintillation, although the characteristic time scale is longer by two to three orders of magnitude. Since the process is random, the errors can be reduced considerably by averaging the results of repeated observations. The uncertainties due to instrumental noise are negligible except for very weak sources.

Atmospheric refraction is not an important source of error in radio astrometry, since the positional information lies in the relative arrival times of the signals at the different antennas rather than in the apparent direction of arrival. Small corrections are necessary to compensate for the slightly differing elevations of the antennas above sea level and for the curvature of the atmosphere, but these are easily calculated and introduce little uncertainty.

The Mullard and NRAO methods both determine declinations absolutely, since in effect they measure the radii of the diurnal circles of the sources. Differences of right ascension between sources are also found absolutely, with an accuracy which is independent of the magnitude of the difference. Unfortunately, the angular sizes 
of the Sun and the planets are too large for their positions to be measured accurately with radio interferometers, and there is therefore no way to refer the right ascensions directly to the vernal equinox by purely radio methods. Instead, it is necessary that the observations include at least one object whose right ascension has been found by optical means. The Mullard right ascensions are referred to the FK4 position of $\beta$ Persei, which is an intermittent radio source (Wade and Hjellming, 1972). At NRAO, the right ascensions have been adjusted to agree in the mean with optical measurements of a large number of sources, mostly quasars (Kristian and Sandage, 1970; Murray et al., 1971).

The major source of systematic error is imperfect instrumental calibration, particularly in the determination of the collimation error (which reflects departures from symmetry in the paths of the signals collected by the different antennas). Small changes in baseline length and orientation due to differing thermal effects at the various antennas can also cause diurnal errors which are difficult to evaluate. Careful calibration procedures can keep such errors small, but they cannot be eliminated entirely.

Accuracies of a few milliseconds in right ascension and a few hundredths of an arc second in declination have been achieved at Mullard (Ryle and Elsmore, 1973). This is fully as good as the FK4. Accuracies of about 0".15 have been attained at NRAO (Brosche et al., 1973). The higher precision of the Mullard measurements is the result of averaging a large number of observations, which much reduces the phase noise caused by atmospheric inhomogeneities. The instruments and methods used at the two observatories are inherently capable of similar accuracy.

\section{Comparison of Radio and Optical Results}

Several fairly extensive lists of accurate optical positions of radio sources have been published in the last 5 years (e.g., Bolton, 1968; Kristian and Sandage, 1970; Hunstead, 1971 ; Véron, 1972; Barbieri et al., 1972). Perhaps the most accurate measurements have been made at Cambridge (Argue and Kenworthy, 1972; Argue et al., 1973). These are essentially on the AGK3 system, although they are based partly on earlier measurements in the FK4 system by Murray et al. $(1969,1971)$.

We shall restrict the present discussion to comparing the Cambridge optical positions with the most recent fundamental radio measurements at Mullard and NRAO (Ryle and Elsmore, 1973; Brosche et al., 1973). Table II gives the measured positions for 1950.0 with their mean errors; the errors of the optical positions are the external errors. The NRAO right ascensions have been adjusted slightly to agree in the weighted mean with the optical values.

It is clear from Table II that the agreement between the three sets of measurements is generally good. This is confirmed by Table III, which gives the weighted (by the inverse squares of the mean errors) average differences between the sets. The overall systematic agreement is at least as good as 0.1 , which is less than the claimed mean errors of the individual NRAO and optical measurements. 
TABLE II

Radio and optical positions (1950.0) for 14 radio sources

\begin{tabular}{|c|c|c|c|c|}
\hline \multirow[t]{2}{*}{ Source } & & \multicolumn{2}{|l|}{ Radio } & \multirow[t]{2}{*}{ Optical } \\
\hline & & Mullard & NRAO & \\
\hline \multirow[t]{2}{*}{$0056-00$} & $00^{\mathrm{h}} 56^{\mathrm{m}}$ & & $31.761 \pm 0.020$ & $31^{s} .771 \pm 0.017$ \\
\hline & $-00^{\circ} 09^{\prime}$ & & $18 . .72 \pm 0 . .52$ & $18.08 \pm 0.30$ \\
\hline \multirow[t]{2}{*}{$3 \mathrm{C} 48$} & $01^{\mathrm{h}} 34^{\mathrm{m}}$ & $49^{s} .827 \pm 0^{s} .002$ & $49^{s} .817 \pm 0^{s} .012$ & $49^{s} .819 \pm 0.012$ \\
\hline & $+32^{\circ} 54^{\prime}$ & $20.63 \pm 0.04$ & $20.74 \pm 0.18$ & $20.40 \pm 0 " .12$ \\
\hline \multirow[t]{2}{*}{$3 \mathrm{C} 84$} & $03^{\mathrm{h}} 16^{\mathrm{m}}$ & $29^{s} .562 \pm 0^{s} .003$ & & $29.548 \pm 0.014$ \\
\hline & $+41^{\circ} 19^{\prime}$ & $51.99 \pm 0.04$ & & $52 " .19 \pm 0 " 12$ \\
\hline \multirow[t]{2}{*}{$3 \mathrm{C} 138$} & $05^{\mathrm{h}} 18^{\mathrm{m}}$ & $16^{s} .526 \pm 0.005$ & $16^{s} .520 \pm 0.011$ & $16.521 \pm 0.011$ \\
\hline & $+16^{\circ} 35^{\prime}$ & $27 " 06 \pm 0.09$ & $27 " .29 \pm 0.25$ & $27.06 \pm 0 \prime .15$ \\
\hline \multirow[t]{2}{*}{$3 \mathrm{C} 147$} & $05^{\mathrm{h}} 38^{\mathrm{m}}$ & $43^{s} .503 \pm 0.003$ & $43.498 \pm 0.016$ & $43^{s} .492 \pm 0.016$ \\
\hline & $+49^{\circ} 49^{\prime}$ & $42^{\prime \prime} .87 \pm 0.02$ & $42^{\prime \prime} .94 \pm 0.15$ & $433^{\prime \prime} 10 \pm 0.12$ \\
\hline \multirow[t]{2}{*}{$0736+01$} & $07^{\mathrm{h}} 36^{\mathrm{m}}$ & & $42^{s} .510 \pm 0.017$ & $42^{s} .496 \pm 0.010$ \\
\hline & $+01^{\circ} 44^{\prime}$ & & $00.29 \pm 0.44$ & $00.29 \pm 0.21$ \\
\hline \multirow[t]{2}{*}{$4 C 39.25$} & $09^{\mathrm{h}} 23^{\mathrm{m}}$ & & $55^{s} .296 \pm 0.014$ & $55^{s} .310 \pm 0.015$ \\
\hline & $+39^{\circ} 15^{\prime}$ & & $24 " .30 \pm 0.17$ & $22.94 \pm 0 " .14$ \\
\hline \multirow[t]{2}{*}{$3 C 279$} & $12^{\mathrm{h}} 53^{\mathrm{m}}$ & & $35.821 \pm 0.021$ & $35^{s} .842 \pm 0.018$ \\
\hline & $-05^{\circ} 31^{\prime}$ & & $07 " .36 \pm 0.30$ & $07.28 \pm 0.30$ \\
\hline \multirow[t]{2}{*}{$3 C 286$} & $13^{\mathrm{h}} 28^{\mathrm{m}}$ & $49^{s} .653 \pm 0.004$ & $49^{s} .681 \pm 0.012$ & $49.657 \pm 0.012$ \\
\hline & $+30^{\circ} 45^{\prime}$ & $58.79 \pm 0.05$ & $58.32 \pm 0.19$ & $58^{\prime \prime} 46 \pm 0.12$ \\
\hline \multirow[t]{2}{*}{$3 C 309.1$} & $14^{\mathrm{h}} 58^{\mathrm{m}}$ & $56^{s} .644 \pm 0.005$ & $56^{8} .616 \pm 0.038$ & $56^{s} .648 \pm 0.033$ \\
\hline & $+71^{\circ} 52^{\prime}$ & $11 " .17 \pm 0.02$ & $11 " .26 \pm 0 " 16$ & $11^{\prime \prime} 33 \pm 0.16$ \\
\hline \multirow[t]{2}{*}{$3 C 345$} & $16^{\mathrm{h}} 41^{\mathrm{m}}$ & $17^{s} .603 \pm 0.002$ & $17^{8} .609 \pm 0^{8} .014$ & $17^{s} .606 \pm 0.013$ \\
\hline & $+39^{\circ} 54^{\prime}$ & $10^{\prime \prime} 89 \pm 0.03$ & $11^{\prime \prime 26} \pm 0.16$ & $10^{\prime \prime} 72 \pm 0.12$ \\
\hline \multirow[t]{2}{*}{ BL Lac } & $22^{\mathrm{h}} 00^{\mathrm{m}}$ & $39: 362 \pm 0^{8} .007$ & $39.370 \pm 0.014$ & $39.397 \pm 0.021$ \\
\hline & $+42^{\circ} 02^{\prime}$ & $08.69 \pm 0.04$ & $08.77 \pm 0.16$ & $08.47 \pm 0.14$ \\
\hline \multirow[t]{2}{*}{ CTA 102} & $22^{\mathrm{h}} 30^{\mathrm{m}}$ & $07^{s} .793 \pm 0.005$ & & $07^{s} .823 \pm 0.029$ \\
\hline & $+11^{\circ} 28^{\prime}$ & $22 " .89 \pm 0.20$ & & $23.07 \pm 0.25$ \\
\hline \multirow[t]{2}{*}{$3 \mathrm{C} 454.3$} & $22^{\mathrm{h}} 51^{\mathrm{m}}$ & $29.510 \pm 0.006$ & & $29 s .533 \pm 0.011$ \\
\hline & $+15^{\circ} 52$ & $54 " .54 \pm 0.09$ & & $54.98 \pm 0.15$ \\
\hline
\end{tabular}

TABLE III

Weighted mean differences

\begin{tabular}{|c|c|c|c|c|}
\hline & Mullard-NRAO & Mullard-Optical & NRAO-Optical & Notes \\
\hline $\begin{array}{l}\langle\Delta \alpha\rangle \\
\langle\Delta \alpha \cos \delta\rangle \\
\langle\Delta \delta\rangle \\
\text { No. of sources }\end{array}$ & $\begin{array}{l}-0^{s} .002 \pm 0.006 \\
-0.01 \pm 0.07 \\
-0.08 \pm 0.09 \\
7\end{array}$ & $\begin{array}{l}-0^{s} .002 \pm 0^{s} .005 \\
-0.01 \pm 0.05 \\
-0.01 \pm 0.08 \\
10\end{array}$ & $\begin{array}{l}- \\
-0.01 \pm 0.05 \\
+0.13 \pm 0.09 \\
10\end{array}$ & $\begin{array}{l}(1) \\
(2)\end{array}$ \\
\hline
\end{tabular}

Notes:

(1) The NRAO right ascensions were adjusted to make the weighted mean difference from the optical equal to zero.

(2) 4C39.25 was omitted because the NRAO and optical declinations differ by 1 ".36, which is excessive in relation to the differences for the other sources. 
The number of sources in Table II is far too small to permit a search for possible regional differences between the radio and optical positions. Even in this small sample, however, there appears to be a consistent trend in the difference between the Mullard and optical right ascensions, as a function of right ascension. This can be seen in Figure 1. The right ascensions of the sources common to the Mullard and optical lists

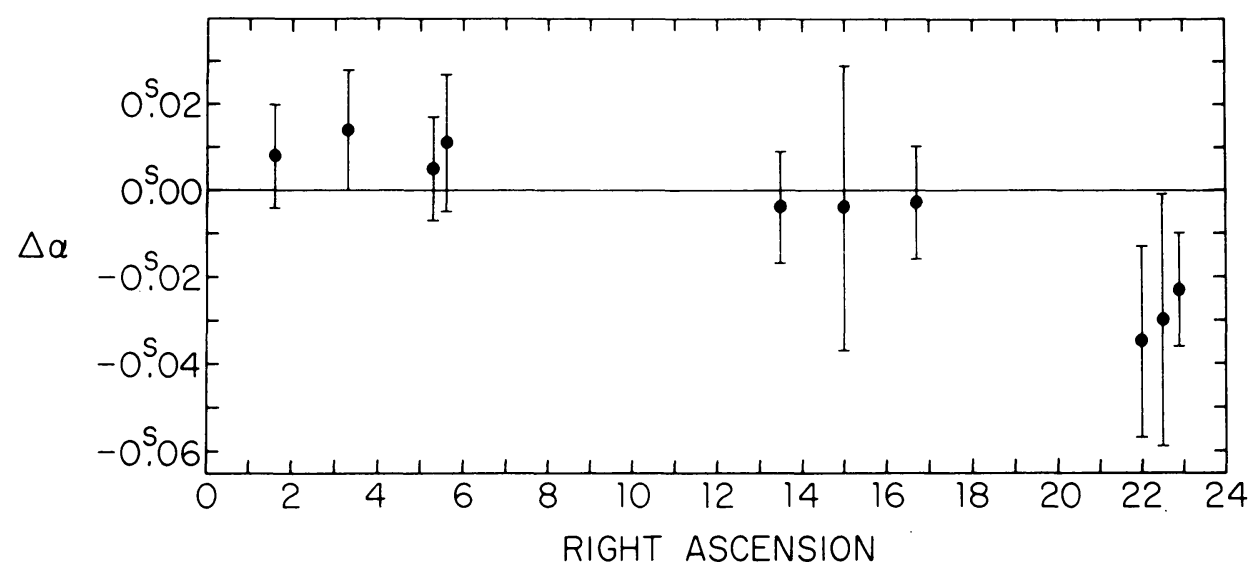

Fig. 1. Distribution of the differences between the Mullard and optical right ascension measurements, as a function of right ascension.

fall into three loose groups. Within each group, the values of $\Delta \alpha$ are about the same, but there is a noticeable difference from group to group. The effect appears to be real, but one cannot say at this time whether the source of the discrepancy is in the optical or the radio measurements. The NRAO measurements are not accurate enough to resolve the matter with any certainty.

\section{Conclusions}

Radio astrometry, although new, has achieved a level of accuracy which rivals the best optical work. It has several valuable advantages over existing optical astrometric methods, of which the most important are:

(a) the relative unimportance of atmospheric refraction;

(b) the ability to measure absolute declinations which are automatically referred to the Earth's instantaneous axis of rotation; and

(c) the ability to measure large angles with essentially the same accuracy as small angles.

The principal disadvantage, from the standpoint of fundamental astrometry, is the inability of existing instruments to determine the location of the vernal equinox without recourse to optical data. This is a temporary deficiency, however, since the Very Large Array (VLA) now under construction in the United States will be sensitive enough to observe the brightest minor planets. 
The major source of random error in radio astrometry is the phase instability caused by tropospheric inhomogeneities. Under average conditions, the accuracy attainable in a single observation with a single pair of antennas is limited to about 0 ". 2 . The error can be reduced considerably by taking the mean of a large number of observations. This is shown by the much higher accuracy of the Mullard measurements in Table II as compared to the NRAO measurements. The Mullard positions are the result of many observations with 16 antenna pairs, while the NRAO positions are derived from single observations (each consisting of $5 \mathrm{~min}$ of integration at three widely separated hour angles) with 3 antenna pairs.

The systematic errors of radio astrometry are due to imperfect instrumental calibration. In a well-designed interferometer system, all of the relevant parameters except the collimation error are highly stable, and most are readily calibratable from radio observations alone. The collimation error is troublesome, however, since it is the result of unstable asymmetries in the electronic system. Its effects can be minimized by careful design of the observing program.

Most of the radio sources which are suitable for astrometric measurement with interferometers are extragalactic, mostly quasars and the nuclei of active galaxies. These objects are numerous and well distributed over the sky. Thus radio astrometry can readily establish a set of precise extragalactic reference points for the differential measurement of proper motions. The faintness of the optical counterparts of most of these objects (15th to 19 th mag.) will cause some difficulty in their practical employment as proper motion standards, but the problem is not fundamental. Finally, repeated measurement of the positions of extragalactic sources over a sufficiently long span of time will permit a refinement of the precessional constants without the complications caused by proper motions and galactic rotation.

Radio observations can make valuable contributions to the field of astrometry as a whole, particularly in establishing a uniform positional reference frame over the entire sky. I believe that radio astrometry will soon play an integral part in the construction of fundamental catalogues, because of its ability to give results which are free of regional systematic errors as well as its inherently high precision. It is unfortunate that there is no radio interferometer in the southern hemisphere at present which is usable for astrometric work. Such an instrument would be invaluable in tying the astrometric systems of the two hemispheres together.

\section{References}

Argue, A. N. and Kenworthy, C. M.: 1972, Monthly Notices Roy. Astron. Soc. $160,197$.

Argue, A. N., Kenworthy, C. M., and Stewart, P. M.: 1973, Astrophys. Letters 14, 99.

Barbieri, C., Capaccioli, M., Ganz, R., and Pinto, G.: 1972, Astron. J. 77, 444.

Bolton, J. G.: 1968, Publ. Astron. Soc. Pacific 80, 5.

Brosche, P., Wade, C. M., and Hjellming, R. M.: 1973, Astrophys. J. 183, 805.

Elsmore, B. and Mackay, D. C.: 1969, Monthly Notices Roy. Astron. Soc. 146, 361.

Hogg, D. E., Macdonald, G. H., Conway, R. G., and Wade, C. M.: 1969, Astron. J. 74, 1206.

Hunstead, R. W.: 1971, Monthly Notices Roy. Astron. Soc. 152, 177.

Kristian, J. and Sandage, A. : 1970, Astrophys. J. 162, 391. 
Murray, C. A., Tucker, R. H., and Clements, E. D. : 1969, Nature 221, 1229.

Murray, C. A., Tucker, R. H., and Clements, E. D.: 1971, Roy. Obs. Bull., No. 162.

Ryle, M.: 1972, Nature 239, 435.

Ryle, M. and Elsmore, B.: 1973, Monthly Notices Roy. Astron. Soc., in press.

Smith, J. W.: 1971, Nature Phys. Sci. 232, 150.

Véron, M. P.: 1972, Astron. Astrophys. 20, 471.

Wade, C. M.: 1970, Astrophys. J. 162, 381.

Wade, C. M. and Hjellming, R. M.: 1972, Nature 235, 270.

\section{DISCUSSION}

Eichhorn: I am not particularly worried about not all of the error boxes overlapping since the true values of a quantity will be within one sigma in only about two of three cases.

Wade: In fact the rate of agreement within the claimed mean errors is about two thirds, as one would expect.

Eichhorn: I am also happy about the apparent approaching demise of the vernal equinox as a fundamental point in astrometry. The vernal equinox is, after all, defined only by the kinematics - and only very incidentally the dynamics - of the Earth rotation and revolution. It is thus very intimately tied to the Earth and the solar system, and really not at all germane to matters galactic and cosmological.

Van Herk: How well should optical positions coincide with radio sources? It has been said that the position of the radio sources depends on the frequency. Have radio sources to be 'compact' to have the two positions (optical and radio) coinciding?

Wade: It is hard to give a general answer, since the radio sources are highly diverse in their properties. In cases where there is a spectral gradient across the source, the radio position will of course be a function of wavelength. When the spectrum is quite uniform over the source, such effects should be negligible. This is true also when the angular extent of the source is appreciably smaller than the error of the positional measurements.

Baars: Yesterday Prof. Bok urged radio astronomers to find all radio stars. I would like to inform you of a project in which Dutch and German radio astronomers use the 100-m telescope in Bonn to search for radio radiation at $3 \mathrm{~cm}$ wavelength. After a possible detection an accurate position is determined with the Synthesis Radio Telescope in Westerbork at $6 \mathrm{~cm}$ wavelength. Several stars have been detected in Bonn, while the Westerbork follow-up is now being made.

I have one new radio star to report, detected by Dr H. Wendker (Hamburg Observatory) and myself with the Westerbork telescope. It is P Cygni and it shows a flux density at $6 \mathrm{~cm}$ wavelength of $10^{-28} \mathrm{Wm}^{-2}$ $\mathrm{Hz}^{-1}$. The derived radio position lies within 0 " 6 from the AGK3 position of the star. The estimated error in the radio position is \pm 0 ". 5 .

Brosche: As long as radio astrometry is using the present constant of precession, 'artificial' proper motions have to be introduced also for extragalactic objects due to the necessary correction of this constant. 